\title{
The genetics of drug-related movement disorders, an umbrella review of meta-analyses
}

Citation for published version (APA):

van der Burg, N. C., Al Hadithy, A. F. Y., Van Harten, P. N., van Os, J., \& Bakker, P. R. (2020). The genetics of drug-related movement disorders, an umbrella review of meta-analyses. Molecular Psychiatry, 25(10), 2237-2250. https://doi.org/10.1038/s41380-020-0660-5

Document status and date:

Published: 01/10/2020

DOI:

10.1038/s41380-020-0660-5

Document Version:

Publisher's PDF, also known as Version of record

Document license:

Taverne

\section{Please check the document version of this publication:}

- A submitted manuscript is the version of the article upon submission and before peer-review. There can be important differences between the submitted version and the official published version of record.

People interested in the research are advised to contact the author for the final version of the publication, or visit the DOI to the publisher's website.

- The final author version and the galley proof are versions of the publication after peer review.

- The final published version features the final layout of the paper including the volume, issue and page numbers.

Link to publication

\footnotetext{
General rights rights.

- You may freely distribute the URL identifying the publication in the public portal. please follow below link for the End User Agreement:

www.umlib.nl/taverne-license

Take down policy

If you believe that this document breaches copyright please contact us at:

repository@maastrichtuniversity.nl

providing details and we will investigate your claim.
}

Copyright and moral rights for the publications made accessible in the public portal are retained by the authors and/or other copyright owners and it is a condition of accessing publications that users recognise and abide by the legal requirements associated with these

- Users may download and print one copy of any publication from the public portal for the purpose of private study or research.

- You may not further distribute the material or use it for any profit-making activity or commercial gain

If the publication is distributed under the terms of Article $25 \mathrm{fa}$ of the Dutch Copyright Act, indicated by the "Taverne" license above, 


\title{
The genetics of drug-related movement disorders, an umbrella review of meta-analyses
}

\author{
Nadine C. van der Burg ${ }^{1,2} \cdot$ Asmar F. Y. Al Hadithy $^{3} \cdot$ Peter N. van Harten ${ }^{1,4} \cdot$ Jim van Os $s^{4,5,6} \cdot$ P. Roberto Bakker ${ }^{1,4,5}$
}

Received: 10 September 2018 / Revised: 10 October 2019 / Accepted: 17 January 2020 / Published online: 4 February 2020

(c) The Author(s), under exclusive licence to Springer Nature Limited 2020

\begin{abstract}
This umbrella review investigates which genetic factors are associated with drug-related movement disorders (DRMD), in an attempt to provide a synthesis of published evidence of candidate-gene studies. To identify all relevant meta-analyses, a literature search was performed. Titles and abstracts were screened by two authors and the methodological quality of included meta-analyses was assessed using 'the assessment of multiple systematic reviews' (AMSTAR) critical appraisal checklist. The search yielded 15 meta-analytic studies reporting on genetic variations in 10 genes. DRD3, DRD2, CYP2D6, HTR2A, COMT, $H S P G 2$ and SOD2 genes have variants that may increase the odds of TD. However, these findings do not concur with early genome-wide association studies. Low-power samples are susceptible to 'winner's curse', which was supported by diminishing meta-analytic effects of several genetic variants over time. Furthermore, analyses pertaining to the same genetic variant were difficult to compare due to differences in patient populations, methods used and the choice of studies included in metaanalyses. In conclusion, DRMD is a complex phenotype with multiple genes that impact the probability of onset. More studies with larger samples using other methods than by candidate genes, are essential to developing methods that may predict the probability of DRMD. To achieve this, multiple research groups need to collaborate and a DRMD genetic database needs to be established in order to overcome winner's curse and publication bias, and to allow for stratification by patient characteristics. These endeavours may help the development of a test with clinical value in the prevention and treatment of DRMD.
\end{abstract}

\section{Background}

Treatment with antipsychotics (AP) can provoke drugrelated movement disorders (DRMD) (also known as extrapyramidal symptoms or EPS), i.e. tardive dyskinesia

Nadine C. van der Burg

nadinevanderburg@yahoo.com

1 Zon \& Schild, GGZ Centraal, Amersfoort, The Netherlands

2 Department of Psychiatry, Amsterdam UMC, Amsterdam, Netherlands

3 Department of Clinical Pharmacy, Parnassia Groep, The Hague, The Netherlands

4 Department of Psychiatry and Psychology, School for Mental Health and Neuroscience (MHeNS), Maastricht University Medical Centre, Maastricht, The Netherlands

5 Department Psychiatry, Brain Centre Rudolf Magnus, Utrecht University Medical Centre, Utrecht, The Netherlands

6 Department of Psychosis Studies, King's College London, King's Health Partners, Institute of Psychiatry, London, UK
(TD), Parkinsonism, akathisia, and acute or tardive dystonia. Dyskinesias are hyperkinetic choreiform involuntary movements which often fluctuate in severity [1]. Parkinsonism is clinically similar to Idiopathic Parkinson disease with the following core features: tremor, rigidity, bradykinesia [1], and postural instability [2]. Akathisia [2] is both a subjective inner feeling of restlessness and objective motor (leg) movements. Dystonia is defined as a syndrome of sustained muscle contraction, frequently causing twisting and repetitive movements or abnormal postures [3].

DRMD can be a reason for non-compliance increasing the risk of a psychotic relapse $[4,5]$ and some DRMD may become irreversible [6]. Chronic patients chronically exposed to AP, particularly when residing in institutional settings, where drug-adherence monitoring is high and polypharmacy is common [7].

The prevalence of DRMD amongst patients varies depending on the type of patient monitored and the rating scales used, as well as interobserver differences. The prevalence is lower in younger patients (32\%) [8], but substantial in chronic patients $(68 \%)[9,10]$, of which a quarter has two types of DRMD [9]. The risk of DRMD is related 
to: (i) the type of AP, dosage and cumulative exposure [1], and (ii) patient-related factors as sex, age, ethnicity, and smoking [1, 10-15]. Several meta-analyses showed a reduced risk of DRMD in second-generation antipsychotics (SGAs), but not their expected disappearance $[6,16]$.

DRMD can cause severe impairment in quality of life [17] and may interfere with daily activities and integration into society through shame, anxiety and social withdrawal $[18,19]$. Also, a meta-analysis [20] and two recent studies showed a higher mortality in patients with tardive dyskinesia $[21,22]$.

Pharmacogenetic research works on prevention strategies by studying different genetic associations with a particular disorder, bridging the gap between pharmacology and genetics. Population-based association studies examine genes that may play a role in the aetiology of a disorder. These 'candidate genes' are selected on a theoretical basis. For example, high sensitivity for the dopamine-D3 receptor (DRD3), which is selectively expressed in a brain region implicated in locomotor function, might be of influence on DRMD [23, 24]. Using a case-control design, associations can be revealed through examination of the allelic distribution in candidate genes.

Since 2005 genome-wide association studies (GWAS) [25] have been developed which have the advantage of a 'hypothesis free' and unbiased approach for examining new DNA variants, which can clarify unknown pathophysiological mechanisms. Several GWAS papers have been published on DRMD identifying promising genes [26-30], which do not concur with the candidate genes that have been studied.

Pharmacogenetic studies may identify genetic risk factors for DRMD [31, 32], paving the way for individually tailored pharmacotherapy [33]. To date, numerous papers have been written on the subject, with inconsistent results [34-36].

An umbrella review (UR) compares and contrasts findings the highest level of evidence i.e. reviews (including meta-analyses), provides a robust synthesis of published evidence and considers the importance of effects found over time, which may be attributed to the winner's curse [37, 38]. Furthermore, the pooled data of meta-analyses may increase the power with more precise estimates.

Given the above considerations, this UR aims to provide clinicians and healthcare-decision makers with evidencebased information about candidate genes of DRMD, recommendations for future prevention and research, and a clear understanding of a broad topic area, in less time than would otherwise be required.

The current UR represents a systematic review of metaanalyses of the candidate genes which are associated with DRMD, updated over time to account for any possible winner's curse [37, 38].

\section{Research objective}

To investigate which genetic factors are associated with DRMD, weighing their effect over time.

A study protocol was registered on PROSPERO (study id CRD42017069634) before the reviewing process commenced [39].

\section{Methods}

\section{Search strategy}

\section{Choice of candidate genes}

We considered genes related to: (i) schizophrenia and dysregulation of the dopamine system because DRMD can be associated with schizophrenia [34, 40], (ii) the metabolism of AP because they are partly responsible for DRMD [41, 42], and (iii) to oxidative stress as DRMDs are related to damage of the dopamine receptor [34, 43, 44]. The following genes were considered for meta-analyses:

\section{Drug metabolism genes}

Cytochrome P450 enzymes CYP2D6, CYP1A2, CYP2C19 and CYP3A4 degrade AP through hydroxylation in the liver. CYP2D6 and CYP1A2 have been studied the most. Within CYP2D6 Alleles 2*, 3*, 4*, 5*, 6*, 7*, 10*, 12*, $14^{*}$, and $41 \mathrm{~B}^{*}$ have been researched in meta-analyses, as well as $* 1 \mathrm{~F}$ and $* 1 \mathrm{C}$ in CYP1A2. Both genes are genetically polymorphic and the allele variations result in different Cytochrome-P450-enzyme activity: lower activity leads to higher drug serum concentrations with potential risk for dose-dependent side effects; and higher activity leads to lower serum concentration with lower drug-response effects. CYP2D6 exhibits various metabolic capacities resulting in patients being poor-, intermediate-, extensiveand ultra-rapid metabolizers (PM/IM/EM/UM). PM are people with two inactive CYP2D6 alleles, IM are homozygous or heterozygous for reduced function alleles $(* 9$, $* 10, * 14 \mathrm{~B}, * 17, * 29, * 41)$, EM with two wildtype alleles (or combinations with $* 2, * 33, * 35, * 39$ alleles) and UM with multiple copies of certain functional alleles $(1, * 2$, *35). The CYP1A2 enzyme metabolises several frequently prescribed SGAs and is seriously inducible by smoking.

\section{Dopaminergic genes}

The most consistent associations in candidate-gene studies of DRMD are observed for the dopamine D3 (DRD3) and D2 (DRD2) receptor genes $[40,45]$. The dopaminesupersensitivity hypothesis proposed that the continuous 
Table 1 Search

Database Search terms

Number of articles

Medline (exp pharmacogenetics, exp clinical genetics, exp medical genetics, exp human genetics, exp molecular genetics, exp gene, exp allele, exp genetics, exp genotype, exp genetic polymorphism, exp single nucleotide polymorphism, exp genetic association, exp nucleotide sequence, single nucleotide.mp, exp gene frequency, exp gene locus, exp genetic code, genetic research.mp, exp genetics, exp genetic association study/ or exp genetic polymorphism/ or exp genetic marker, genetic testing.mp, exp genetic database, exp genetic background, exp genetic predisposition, exp genetic load, exp gene frequency, exp genotype environment interaction, exp genetic linkage, exp genome-wide association study, exp DNA, exp genome, exp whole genome sequencing, whole genome.mp, gwas.mp, exp gene expression/ or exp epigenetics/ or exp epigenesis, exp whole genome sequencing, WGS.mp.) AND (basal ganglia disease.mp, exp extrapyramidal syndrome, exp dopamine, exp extrapyramidal system, exp brain, exp corpus striatum, movement disorder.mp, exp motor dysfunction, exp tardive dyskinesia, exp parkinsonism, exp akathisia, exp dystonia, tardive dyskinesia.mp, dystonia.mp, akathisia.mp, parkinsonism.mp.) AND (adverse effect.mp, exp adverse drug reaction, exp drug therapy, therapeutics.mp, exp drug induced disease, neuroleptic induced.mp, exp atypical antipsychotic agent/ or antipsychotic induced.mp, typical antipsychotic.mp, first generation antipsychotic.mp, second generation antipsychotic.mp, antipsychotic.mp, neuroleptic.mp, drug induced.mp, exp drug toxicity, drug related side effects.mp, exp side effect, exp chemically induced disorder, adverse drug reaction systems.mp, drug related.mp, psycho*.mp, psychotic disorder.mp. or exp Psychotic Disorders, exp Bipolar Disorder/ or exp Schizophrenia/ or schizo*.mp, bipolar.mp) AND (meta analyses.mp, exp Meta-Analysis, meta analysis.mp.)

Embase (exp pharmacogenetics, exp clinical genetics, exp medical genetics, exp human genetics, exp molecular genetics, exp gene, exp allele, exp genetics, exp genotype, exp genetic polymorphism, exp single nucleotide polymorphism, exp genetic association, exp nucleotide sequence, single nucleotide.mp, exp gene frequency, exp gene locus, exp genetic code, genetic research.mp, exp genetics, exp genetic association study, exp genetic polymorphism, exp genetic marker, genetic testing.mp, exp genetic database, exp genetic background, exp genetic predisposition, exp genetic load, exp gene frequency, exp genotype environment interaction, exp genetic linkage, exp genome-wide association study, exp DNA, exp genome, exp whole genome sequencing, whole genome.mp, gwas.mp, exp gene expression, exp epigenetics, exp epigenesis, exp whole genome sequencing, WGS.mp) AND (basal ganglia disease.mp. or exp extrapyramidal syndrome, exp dopamine, exp extrapyramidal system, exp brain, exp corpus striatum, movement disorder.mp, exp motor dysfunction, exp tardive dyskinesia, exp parkinsonism, exp akathisia, exp dystonia, tardive dyskinesia.mp, parkinsonism.mp, akathisia.mp, dysthonia.mp) AND

(adverse effect.mp. or exp adverse drug reaction, exp drug therapy, therapeutics.mp, exp drug induced disease, neuroleptic induced.mp, exp atypical antipsychotic agent, antipsychotic induced.mp, typical antipsychotic.mp, first generation antipsychotic.mp, second generation antipsychotic.mp, antipsychotic.mp, neuroleptic.mp, drug induced.mp, exp drug toxicity, drug related side effects.mp, exp side effect, exp chemically induced disorder, adverse drug reaction systems.mp, drug related.mp, exp Bipolar Disorder, bipolar.mp, exp Psychotic Disorders, exp Schizophrenia, schizo*.mp, psycho*.mp) AND (meta analysis, meta analys\#s.mp)

Psychinfo (exp Genetics, pharmacogenetics.mp, clinical genetics.mp, medical genetics.mp, human genetics.mp, molecular genetics.mp, exp GENES, gene.mp, exp ALLELES, allele.mp, genotype.mp, exp GENOTYPES, genetic polymorphism.mp, single nucleotide polymorphism.mp, genetic association.mp, nucleotide sequence.mp, single nucleotide.mp, gene frequency.mp, gene locus.mp, genetic code.mp, genetic research.mp, genetic association study.mp, genetic polymorphism.mp, genetic marker.mp, exp Genetic Testing, genetic database.mp, genetic background.mp, genetic predisposition.mp, genetic load.mp, gene frequency.mp, genotype environment interaction.mp, exp Genetic Linkage, genome wide association study.mp, exp DNA, exp GENOME, whole genome sequencing.mp, whole genome.mp, gwas.mp, exp Gene Expression, whole genome sequencing.mp, WGS.mp, exp epigenetics, exp epigenesis)

AND (exp Basal Ganglia, exp Movement Disorders, basal ganglia disease.mp, exp Extrapyramidal Tracts, exp Extrapyramidal Symptoms, extrapyramidal syndrome.mp, exp DOPAMINE, extrapyramidal system.mp, exp BRAIN, corpus striatum.mp, exp Basal Ganglia, exp Movement Disorders, motor dysfunction.mp, exp Tardive Dyskinesia, exp PARKINSONISM,exp AKATHISIA, dystonia.mp, tardive dyskinesia.mp, parkinsonism.mp, akathisia.mp, akathisia.mp)

AND (exp Drug Therapy, exp "Side Effects (Drug)", adverse effect.mp, therapeutics.mp, drug induced disease. $\mathrm{mp}$, exp Neuroleptic Drugs, neuroleptic induced.mp, atypical antipsychotic agent.mp, antipsychotic induced.mp, typical antipsychotic.mp, first generation antipsychotic.mp, second generation antipsychotic.mp, anti psychotic. $\mathrm{mp}$, neuroleptic.mp, drug induced.mp, drug toxicity.mp, related side effects.mp, chemically induced disorder. $\mathrm{mp}$, adverse drug reaction systems.mp, drug related.mp, psycho*.mp, exp Schizophrenia, exp Bipolar Disorder, psychotic disorder.mp, schizo*.mp, bipolar.mp) AND (meta analysis.mp, exp Meta Analysis, meta analyses.mp)

occupation of dopamine receptors results in an upregulation of dopamine receptors. This occurs in the basal ganglia including the extrapyramidal system, making the patient susceptible for DRMD [40]. The DRD3 contains an SNP that substitutes serine (Ser) into glycine (Gly) (Ser9Gly polymorphism, rs6280). Patients with Gly-Gly 
homozygosity or heterozygotes have a four times higher affinity for dopamine3 ligand (D3 ligand) than Ser-Ser genotypes [34]. The DRD2 contains three SNPs that have been studied by meta-analysis. The DRD2 TaqlA polymorphism (rs1800497) is located in the bordering in the $A N K K 1$ gene, which is involved in signal transduction [46]. Patients with A1A1 or A1A2 genotypes have a lower D2-receptor count in the striatum compared with A2 heterozygotes. DRD2 -141C Ins/del (rs1799732) has been reported to reduce DRD2 promotor activity in vitro and an increase in striatal D2 binding in vivo [40], while Ser311Cys (rs1801028) affects intracellular signalling [47].

Dopamine activity is terminated by dopamine reuptake transporters and by metabolic degradation via catechol- $O$ methyltransferase (COMT). The COMT gene contains an SNP (Val158Met, rs4680) and it leads to valine changing to methionine [34]. The met allele results in a less active and less stable enzyme and therefore more dopamine stays present in the synapse. Due to the high levels of dopamine in the prefrontal cortex, negative feedback results in lower dopamine levels in other parts of the brain. Whether COMT influences the risk of TD by higher free radical formation or postsynaptic dopamine receptor super sensitivity is not yet known [40].

\section{Serotoninergic genes}

Serotonin plays an important role in dopaminergic neurotransmission regulation and genetic variation and the gene encoding the 5-HT2A receptor (5HTR2A) may impact DRMD. 5-HT2A receptors in the striatum and substantia nigra exert an inhibitory effect on dopamine in the nigrostriatal pathway, which could result in a decrease of DRMD, in patients on AP. This could explain why some SGAs, which act more on serotonergic neurons, may have a lower potency for TD [43]. Of the HTR2A SNPs, 102T/C (rs6313) and His452Tyr (rs6314) are the most researched.

\section{Oxidative stress genes}

Oxidative stress and free radicals play a significant role in oxidative damage and cell death and can occur when using AP [43]. Using AP, repeated exposure to free radicals occurs while the antioxidant system is not sufficiently equipped to counteract this, causing permanent neuronal damage. This hypothesis is known as the "neuronal degeneration hypothesis" [48].

Superoxide-dismutase-2 gene (SOD2) codes for superoxide dismustase enzyme which is involved in mitochondrial oxidative metabolism [34]. SOD2 is an enzyme that prevents the formation of free radicals [33]. The SOD2 contains a functional SNP (Ala9Val, rs4880) affecting
SOD2 activity. Contrary to expectation, the less effective Val allele was found to be protective for TD [34]. NADPH (quinine oxidoreductase 1:NQO1) is also involved in the detoxification of superoxide radicals and carries a functional polymorphism (Pro187Ser, rs1800566). Compared with the Pro/Pro-wildtype patients with the Ser/Ser genotype hardly produce any NQO1 protein $(2-4 \%)$.

\section{Other genes}

\section{Brain-derived neurotropic factor gene (BDNF)}

BDNF maintains and promotes DRD3 expression [49]. It positively affects neuronal growth, survival, and differentiation [50]. Several studies have shown that schizophrenia patients with TD had lower plasma BDNF than patients without TD [49]. BDNF contains an SNP (rs6265), which has been reported to affect human episodic memory and neuronal function [51].

\section{HSPG2 gene}

The HSPG2 gene encodes perlecan, which binds to various basement membrane proteins, such as collagen IV and to cell-surface receptors [52]. The HSPG2 rs2445142 $\mathrm{G}$ allele was found to be the risk allele for TD in a GWAS on 86 Japanese schizophrenia patients with treatment-resistant TD. This allele was also associated with an increase in gene expression in human prefrontal cortical tissues. HSPG2 deficiency led to fewer vacuous chewing movements in a mouse model of TD [53].

\section{Search}

Medline, Embase and Psychinfo were searched from inception up to 23-09-2019 using various search terms and their synonyms (Table 1). Titles and abstracts were scanned for potentially eligible reviews by two authors (NB, PRB), before retrieving full articles. Results without "meta-analysis" in the title or abstract were excluded. No language restrictions were applied. Conflicting judgements were resolved by discussion to reach consensus, otherwise checked by a third reviewer $(\mathrm{AH})$. Two review authors (NB, PRB) hand searched the reference lists of the relevant articles retrieved (Fig. 1).

\section{Critical appraisal}

Two review authors (NB, PRB) assessed the methodological quality of included meta-analyses, using the 'assessment of multiple systematic reviews' (AMSTAR) critical appraisal checklist [54]. The AMSTAR instrument assesses the quality of systematic reviews and meta-analyses. 
Fig. 1 Flowchart.

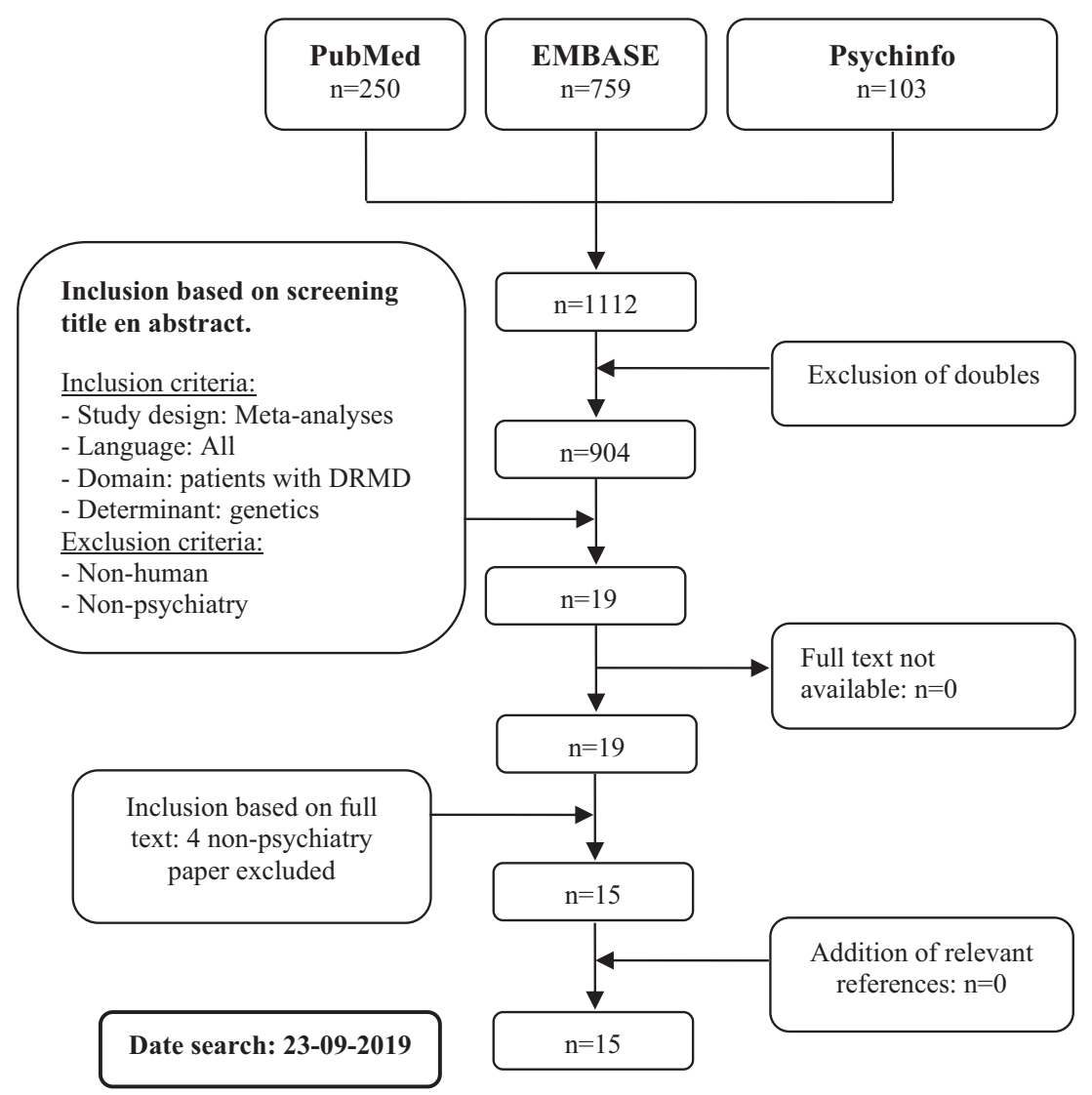

\section{Results}

The search yielded 15 meta-analytic studies and consensus was obtained. No additional studies were found by crossreferencing (Table 2). The meta-analyses found, considered various SNPs in 10 candidate genes related to TD: $D R D 2$, DRD3, HTR2A, CYP1A2, CYP2D6, SOD2, COMT, NQO1, $B D N F$ and $H S P G 2$. One meta-analysis [41] also included parkinsonism, akathisia and acute dystonia in addition to TD in the analysis.

\section{Drug metabolism genes}

Patsopoulos et al. [42] demonstrated, with a meta-analysis of eight studies on CYP2D6 and TD, a moderate effect of any loss of function (LF) alleles on TD (odds ratio $(\mathrm{OR}=$ 1.43)). LF alleles were grouped owing to the major effect on the function of the protein, and to the sample size, in which comparing the relative risk between alleles was not possible. Seven of the eight studies examined white patients. Subjects received a variety of AP, including both first-generation AP (FGAs) and SGAs. Information on sex distribution was available in only three studies. Three out of eight studies were not in Hardy-Weinberg Equilibrium (HWE) $(P=0.03, P=0.001$ and $P<0.001$, respectively) and they were excluded after sensitivity analyses. The
Hardy-Weinberg law states that in a large random mating population at equilibrium genotype frequencies are functions of allele frequencies, and that genotype frequencies can be predicted by allele frequencies. Therefore significant deviations from HWE predictions could stem from population stratification, selection bias and genotyping errors. If HWE is violated, the inferences of these studies may thus be biased [55]. Heterogeneity was not present, and no check for publication bias was performed (Table 2). Heterozygotes for CYP2D6 LF alleles and thus PM had an OR of 1.50 for TD compared with wildtype (Wt) homozygotes (Wt/Wt). The summary OR had been stable and not changing in the same direction over time. There was evidence that the strength of the association between TD and LF alleles was more prominent in small studies, whereas it was diminished in larger studies. Another meta-analysis of four studies on TD did not find an increase of risk for TD, except when only prospective studies were considered, showing that heterozygotes had an increased odds for TD $(\mathrm{OR}=2.08)$ when compared with the wild type/wild type genotype, i.e. alleles $* 1$ and $* 2$ (Table 2) [41]. In this study, alleles were also considered functional (wild type) or mutant and metaboliser status was not distinguished. Incomplete data on HWE was given with two out of four studies checking for HWE and publication bias was not mentioned. There was no evidence for heterogeneity. These 


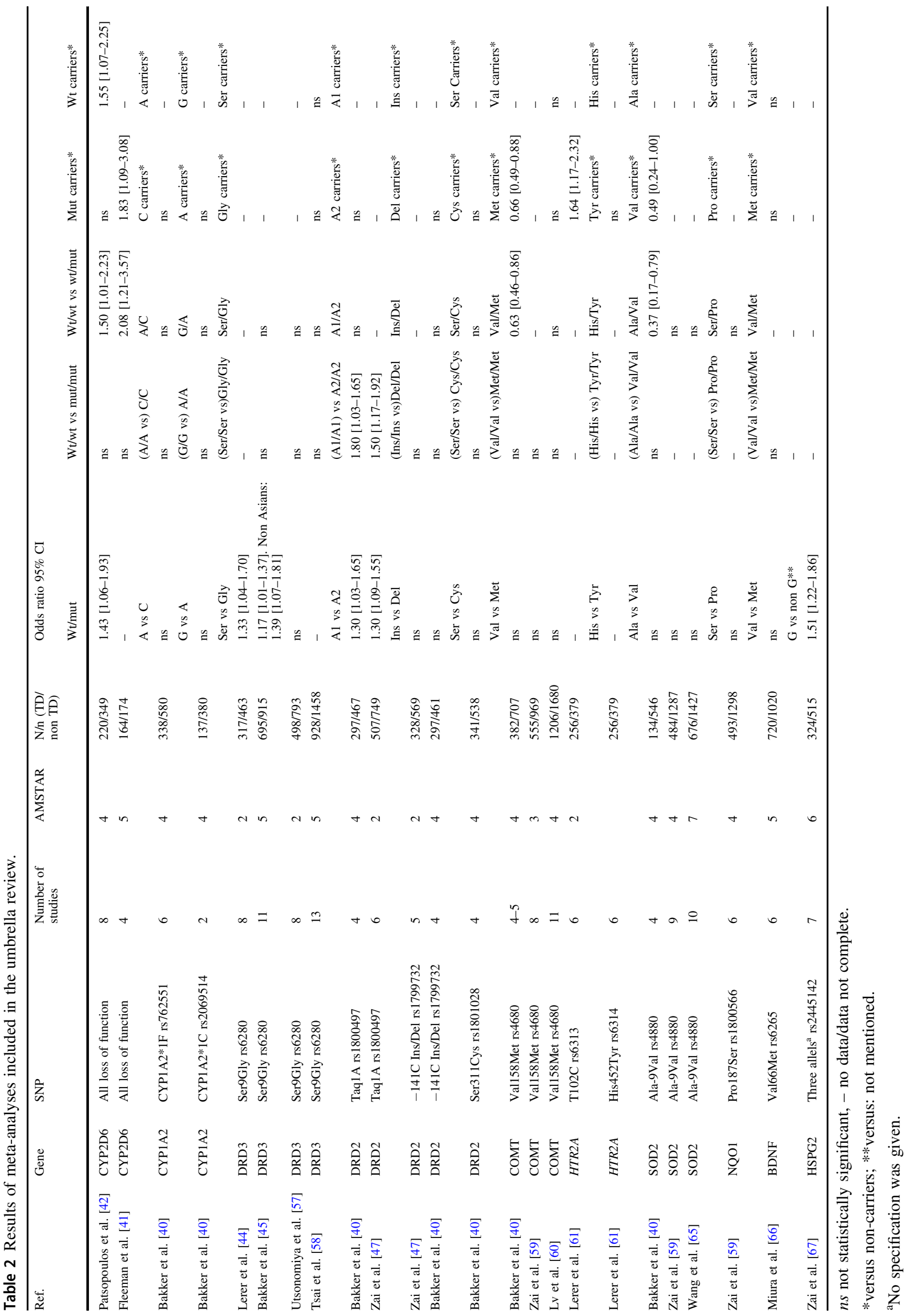


four studies had also been included by Patsopoulos et al. [42].

Three out of four abovementioned studies had a small sample size (26 TD patients or less). One study included 110 Asian patients with TD. Thus, most patients in the meta-analyses were of Asian descent. Subjects received FGAs or SGAs or both (Table 3). Patients who were mutation carriers had an OR of 1.83 compared with $\mathrm{Wt} / \mathrm{Wt}$. Patients who had a wild type/mutation (Wt/mut) CYP2D6 genotype were more likely to develop parkinsonism than patients with wild type/wild type (Wt/Wt) genotype $(\mathrm{OR}=$ 1.64). No association between $C Y P 2 D 6$ and akathisia or acute dystonia was found.

The CYP1A2 enzyme metabolises SGAs clozapine and olanzapine and is inducible by smoking. $C Y P 1 A 2$ contains two studied SNPs: $C Y P 1 A 2 * 1 \mathrm{~F}$ and $C Y P 1 A 2 * 1 \mathrm{C}$. In the analyses of $C Y P 1 A 2 * 1 \mathrm{~F}$ six studies were included, four out of six studies included Asians only [40]. The type of AP administered and sex of subjects were not mentioned. No publication bias or heterogeneity was present (Table 3). It was not mentioned whether the study groups were in HWE. Bakker et al. [40] found no increased risk for TD for the $C Y P 1 A 2 * 1 \mathrm{~F}$ polymorphism even when the groups were confined to smokers, Asians or both; Asian are known to have lower CYP1A2 activity (Table 2) [40]. In the CYPA2*1C meta-analyses two studies were included, containing all Asian subjects. Heterogeneity and/or publication bias was not mentioned. Bakker et al. [40] found no increase risk in for the $C Y P 1 A 2$ gene when studying SNP CYPA2*1C (Table 2) [40].

\section{Dopamine system genes}

A meta-analysis [56] pooled eight studies on DRD3 (Ser9Gly polymorphism, rs6280), with $97 \%$ white subjects, yielding a pooled OR of 1.33, indicating moderately increased risk of developing TD (Table 2). In 23 of 317 patients TD was assessed clinically. For two study groups of patients in the meta-analyses, only data of abstract could be used. The type of AP was not mentioned and the meta-analysis had a low AMSTAR score. HWE was checked and the TD groups from Jerusalem and Nithsdale, were not in HWE. There was no heterogeneity, or publication bias. (Table 3). Bakker et al. [45] calculated a pooled OR of 1.17 for carrying the Gly allele and TD, in 12 studies (Table 2). In total 7 studies included only Asian subjects (Table 3). Bakker et al. conducted an ethnic stratified, meta-analysis describing in carriers of the Gly allele an OR of 1.07 for TD in all studies including Asians, and 1.39 in non-Asians [45]. HWE was checked: one study was not in equilibrium, whilst another did not report on HWE. There was no heterogeneity, or publication bias. Similarly, a meta-analysis of eight studies in Asians [57] found no significant effect on OR for TD (Table 2).
Utsunomiya et al. [57] excluded four studies with only white subjects and one study with only Jewish subjects included by Bakker et al. [40]. The analysis was adjusted for age and sex. However, the meta-analyses showed a low AMSTAR score of two (Table 3). HWE and publication bias were not checked. A meta-analysis by Tsai et al. [58] pooled data from 13 studies, of which eight conducted with Asians (59\%), showed a non-significant decrease in odds $(\mathrm{OR}=0.93)$ (Tables 2 and 3). Although the meta-analyses of Bakker et al. [45] and Tsai et al. [58] were similar in demographic composition and other important variables, the number of subjects with TD were different, i.e. 695 and 928, respectively. One study was not in HWE. Tsai et al. [58] found a significant publication bias and heterogeneity.

Bakker et al. [40] conducted a meta-analysis of four articles, considering DRD 2 TaqlA SNP (rs1800497), containing 297 TD patients (57\% Asian descent). Using A1/A1 genotype as a reference point, the pooled ORs were 1.80 and 1.15 for A2/A2 and A1/A2 genotypes, respectively, indicating a higher risk for patients with the A2 allele (Table 2). Groups were in HWE; one study did not inform on HWE. MAF differed between the ethnic groups. There was no evidence for heterogeneity or publication bias. A second meta-analysis conducted by Zai et al. [47], with 507 TD subjects in six studies, found a higher $\mathrm{A} 2$ frequency in TD patients (effect size 1.30) and a higher A2/A2 genotype frequency in TD patients (effect size 1.50) compared with patients without TD (Table 2). Ethnicity, age, and sex ratio did not contribute to the results observed (Table 3). Significant heterogeneity or publication bias was not present and HWE was not examined.

Another SNP polymorphism in DRD2 studied in the metaanalysis by Bakker et al. [45] was the Ser311Cys polymorphism (rs1801028). The four studies included 341 cases of TD, with $84 \%$ of patients being Asian (Table 3). A nonsignificant pooled OR was found for the genotypes for Ser311Cys (Table 2). Publication bias or heterogeneity was not observed and HWE was not mentioned in either meta-analysis.

Bakker et al. [40] studied the -141C Ins/Del polymorphism (rs1799732) in four studies consisting of 297 patients, no significant association between TD and this polymorphism was observed (Table 2). All studies used AIMS for observation of TD. A similar meta-analysis by Zai et al. [47] concluded in the same result. Both metaanalyses reported no publication bias or heterogeneity. HWE was not mentioned in either meta-analyses.

\section{Catechol-0-methyltransferase (COMT)}

To date, three meta-analyses have been performed on polymorphism rs4680 (Val158Met). Bakker et al. [40] pooled five studies (382 patients with TD and all Asian) and concluded in an OR of 0.63 of Met-Val genotype when comparing to Val-Val homozygotes $(\mathrm{OR}=0.63)$ (Table 2$)$. 


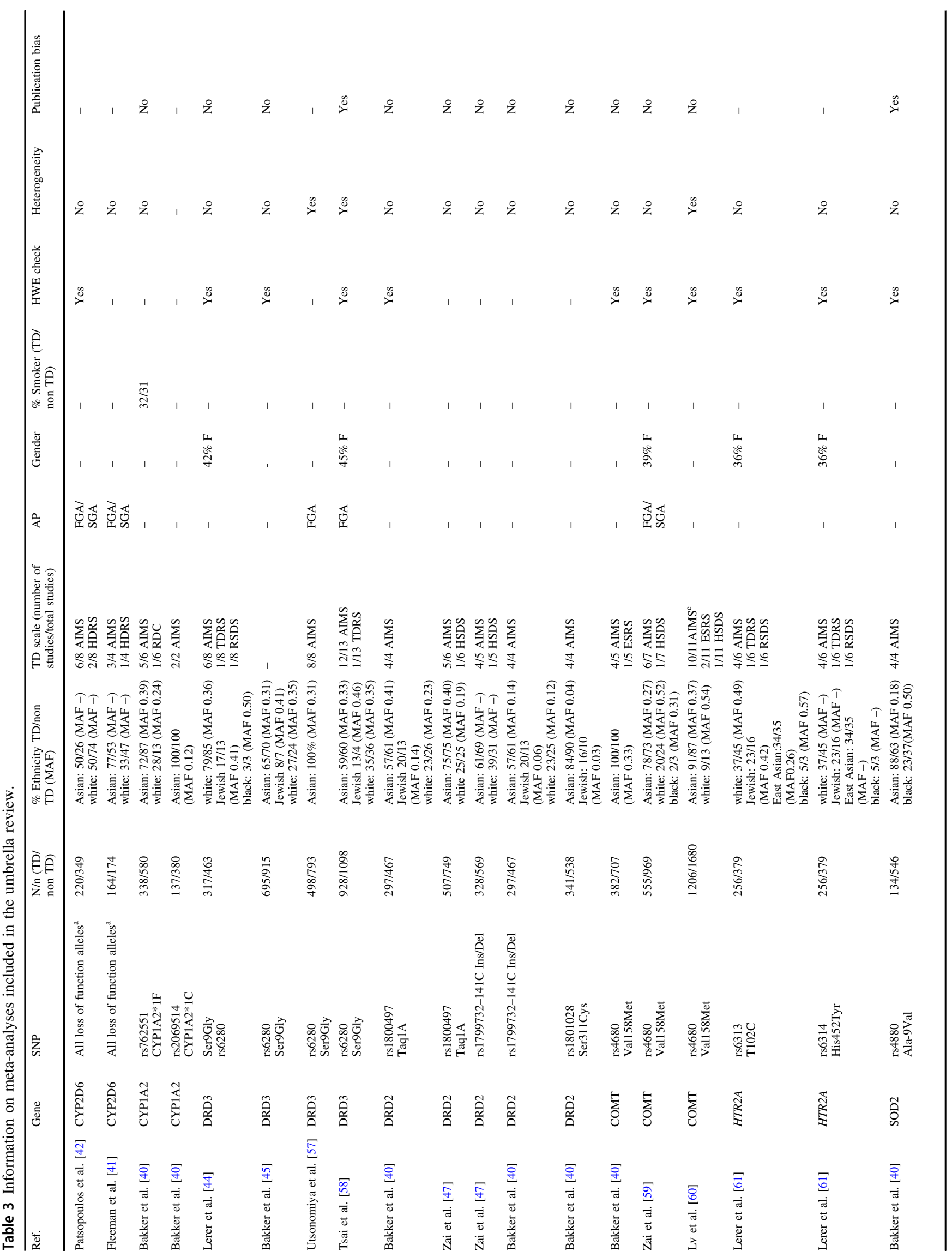




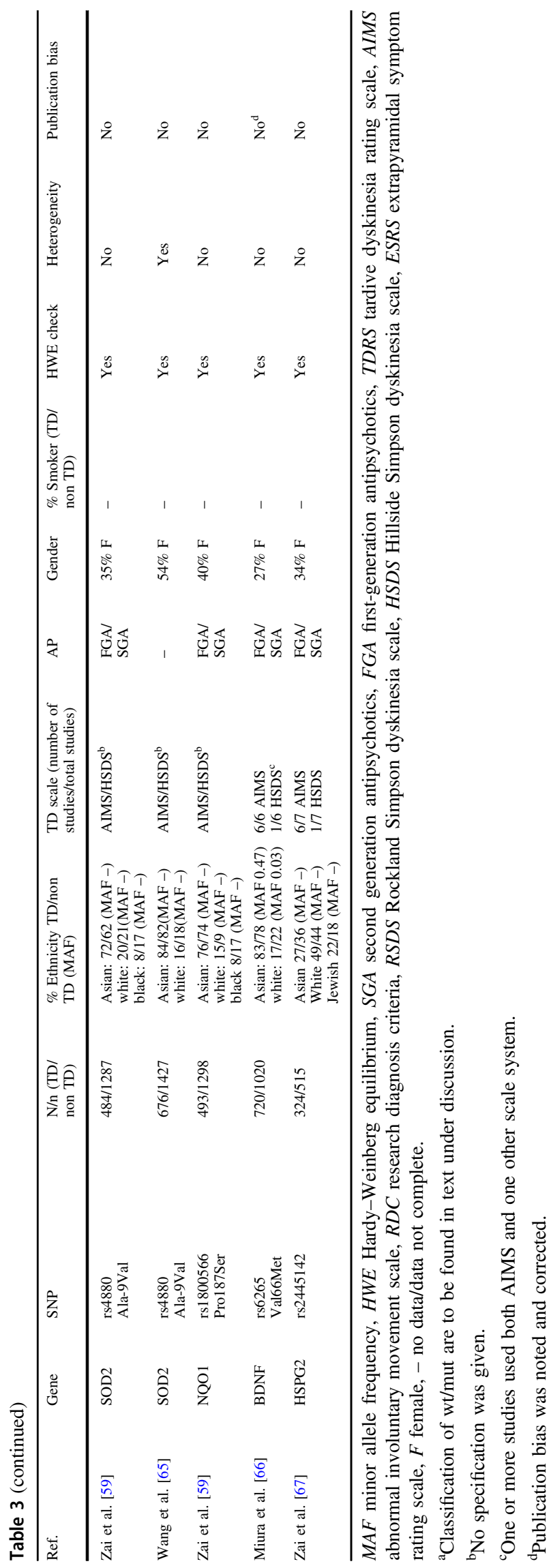

The met allele results in a less active and less stable enzyme and it is hypothesised that patients with the met allele have a higher risk of TD. Three studies were in HWE, one study was not and one study did not report on HWE. No heterogeneity was present in the genotypic comparisons (Table 3). Zai et al. [59] performed a meta-analysis of seven patient groups (553 with TD), including the five articles analysed by Bakker et al. [40] and adding a Korean study and an own present study consisting of white and black subjects. Zai et al. performed a sex-stratified meta-analysis, $39 \%$ being female, and found a significant association between the $\mathrm{Val} / \mathrm{Val}$ genotype and TD occurrence $(\mathrm{OR}=$ 1.63), only in females (Table 2). All but one of the studies were in HWE. There was no evidence for heterogeneity or publication bias. The largest meta-analysis was performed by Lv et al. [60], who included 11 studies (nine of which included only Asian patients) with 1206 TD cases; they included all studies that had been used in the meta-analysis by Zai et al. [59] and Bakker et al. [40]. They additionally included four large Chinese samples. The overall pooled results indicated no significant association between COMT Val158Met gene polymorphism (rs4680) and TD (Table 2). They conducted a subgroup analysis by ethnicity and the conclusion did not change. No publication bias was found. A mild to moderate degree of heterogeneity was present and one study did not report on HWE (Table 3). In all studies, MAF differed between ethnic groups.

\section{Serotonin system genes}

The HTR2A gene T102C (rs6313) SNP is the most extensively researched. A pooled analysis of 635 patients with DRMD in six studied groups showed an OR of 1.64, indicating a significant increase in risk of orofacial TD for patients with a $\mathrm{C}$ allele compared with the $\mathrm{T}$ allele (wild type) [61] (Table 2). This result remained after controlling for age and sex. The T102C genotype was significantly associated with TD in older patients and in patients with non-orofacial TD. One of the six study groups (the largest: containing 88 patients) was not in HWE, publication bias was not analysed and heterogeneity was not present. Another SNP (His452tyr, rs6314) in the same study did not contribute on its own but did become significant when coupled to the T102C SNP [61] (Table 2). The authors stress the importance of considering the SNPs in the context of a panel of polymorphisms associated with DRMD.

\section{Oxidative stress}

A meta-analysis including four studies (134 patients with TD) found a significant overall protective effect of the Val9 polymorphism (rs4880) for TD in the SOD2 gene (OR 0.37 
for Ala-Val heterozygotes) [40] (Table 2). Bakker et al. [40] excluded two studies due to the use of Polish language [62], and not reporting on data [63]. Three out of the four studies consisted of only Asian patients. One study was not in HWE, and there was evidence for overall publication bias (Table 3). However, in a second meta-analysis done by Zai et al. [64], including $484 \mathrm{TD}$ patients in nine study groups, no increased odds were found. The three out of five extra included studies were published after the search by Bakker et al. [40] search was completed. Zai et al. [64] included the studies excluded by Bakker et al. [40] but later excluded these studies due to heterogeneity. The studies included were in HWE, and no publication bias or heterogeneity in the meta-analysis was found after the exclusion of the two studies. The largest meta-analysis concerning SOD2 was done by Wang et al. [65] and included ten studies (7 of which had only Asian patients) with 676 TD patients. Three out of ten studies had not been included in the meta-analysis published by Zai et al. [64]. HWE was checked in all studies (Table 3), but not reported. There was no significant association between Ala9Val alleles and TD (OR 0.90 CI 0.76-1.06) (Table 3). No publication bias was observed, however, heterogeneity was considerable.

Zai et al. [64] meta-analysed six studies including 493 TD patients who were genotyped for NQO1 Pro187Ser (rs1800566). All studies were in HWE. Heterogeneity was found and the oldest study was excluded because it showed an opposite trend to the other later studies. There was no evidence for publication bias. Four out of six studies included Asian patients exclusively (Table 3 ). Ethnicity and sex ratio did not have any influence on the results observed. The allelic, and genotypic results remained insignificant (Table 2).

\section{Other genes}

\section{BDNF}

Miura et al. [66] meta-analysed six studies reporting on Val66Met (rs6265) of BDNF. All studies together encompassed 1740 patients, 720 with TD, 26.9\% female and 83\% Asian (Table 3). There was no significant association between the Val66Met polymorphism and TD when comparing met carriers and non-met carriers (Table 2). MAF differed between the Asian and white groups. All studies were in HWE, and there was no heterogeneity or publication bias (after correction). They performed an-a-priorydefined-subgroup analyses by race, Asians vs. white, which showed no significant increase in TD occurrence. However, a higher AIMS score was found in white subjects who were met allele carriers versus met non-carriers, they consisted of a small group of patients. This was not found with the Asian group.

\section{HSPG2 gene}

A meta-analysis [67] found a significant association of the $\mathrm{G}$ allele (rs2445142) with TD occurrence (OR 1.51 [1.22-1.86]) (Table 2). It was not stated which allele was compared. Because the Taqman assay does not detect $\mathrm{G}$ to A changes we assume the allele in comparison was the $\mathrm{C}$ allele. All studies were in HWE. Much of the appeared effect seemed to originate from the original dataset, which concluded in an OR of 2.25 (1.55-3.27). Six of the seven studies used AIMS criteria and $34 \%$ of patients were female. No heterogeneity or publication bias was found. Carriership frequencies were not stated (Table 3).

\section{Discussion}

\section{Summary of findings}

The current UR provides a synthesis of different published reviews relevant to a specific question. We found 15 metaanalyses of various SNPs in 10 candidate genes of which seven genes (DRD3, DRD2, CYP2D6, HTR2A, COMT, $H S P G 2$, and SOD2), appeared to show a degree of associations with TD, and CYP2D6 with Parkinsonism. Of the seven genes CYP2D6 showed the strongest association with DRMD, followed by DRD2, HTR2A, HSPG2, DRD3, SOD2 and COMT. No suggestive or significant associations were found with akathisia or tardive dystonia.

\section{Specific genetic findings}

Despite differences in methodology size and ethnic composition between the two meta-analyses on CYP2D6 [41, 42], both found a higher risk of TD in subjects with Wt/ mut genotype vs. Wt/Wt genotype and there is evidence that an $\mathrm{LF}$ allele increases the risk of TD. In the Patsopoulos et al. [42] meta-analyses all LF alleles were grouped because of (i) major effect on the function of the enzyme and (ii) sample size, in which comparing the relative risk between the alleles was not possible. In the meta-analysis by Fleeman et al. [41], alleles were also considered to be mutant or Wt. Because the alleles were grouped, the risk of DRMD could not be distinguished in PM/IM/EM/UM in this UR. Patsopoulos et al. [42] found a stronger association between TD and LF alleles in CYP2D6, when the smaller studies included in his meta-analysis were considered. The larger OR in the meta analysis by Fleeman et al. [41], could be explained by the larger amount of small studies, when compared with Patsopoulos et al. [42] meta-analysis. Three out of the four studies included by Fleeman et al. [41] had small sample sizes (26 TD patients or less) which could have strongly influenced the meta-analysis. 
Bakker et al. [40] Is the only one who studied both CYP1A2*1F and CYP1A2*1C alleles (Table 3 ). It is possible that the non-significant results, also after stratification by ethnicity and smoker status, are due to CYP1A2 not impacting the risk of TD and/or low study power.

The 13 studies and four meta-analyses on DRD3 Ser9Gly (rs6280) polymorphism show conflicting results, which could be due to the underlying ethnic differences between the study groups [45, 56-58] (Table 2). An ethnicitystratified meta-analysis by Bakker et al. [45] showed an OR of 1.39 [1.07-1.81] in non-Asians but a non-significant OR in Asians. In Asians, the Gly allele was found to be protective for TD, contrary to the white subjects. This stresses the importance of ethnic stratification in analysis.

Two meta-analyses on DRD2 Taq1A polymorphism (rs1800497), [40, 47] showed similar significant results (Table 2), although they differed methodically (Table 3 ). It is likely DRD2 TaqlA polymorphism influences the risk of TD.

COMT is one of the most researched genes in TD with 11 studies and three meta-analyses [40, 59, 60]. The results provide some support for the hypothesis that the COMT impacts risk of TD, possibly depending on race and sex. Within the three meta-analyses, not all included studies were in HWE, which could influence results of all three meta-analyses by faults as selection bias, population stratification, and genotyping errors. This could explain the mixed findings between the meta-analyses (Table 3) [55]. Zai et al. [59] states that the mixed findings of the different studies on COMT could be due to the possibility that the Val158Met (rs4680) SNP is sex-specific (and estrogen influenced), emphasising the need for sex-specific analyses, not only in TD but also in other psychiatric disorders.

The only meta-analyses on two HTR2A SNPs demonstrated a small increase in TD in C allele carriers of rs6314 SNP and replication is needed. Lerer et al. [61] combined rs6314 to rs6313 (His452Tyr), making the non-significant separate SNPs a significant result in joint contribution. The low MAFs in several ethnic groups of rs6313 would require much larger samples to detect an association. Lerer et al. [61] stressed that combining polymorphisms could increase the knowledge of the polygenetic basis of the complex phenotype of TD.

One meta-analysis was performed on NQO1. [64] and the non-significant results could be due to NQO1 not playing a major role in the pathogenesis of TD or that the sample size was insufficient with the low MAFs in the white and black subjects (Table 3). Zai et al. [64] additionally studied the 9Val polymorphism (rs6314) in SOD2. In contrast to Bakker et al. [40], Zai et al. [64] did not find any significant effect. The disappearance of the significant results could be caused by 'winners curse' [37]. The same inconclusive result was reported in the next large meta-analysis [65], however, heterogeneity in this study was high, which could have influenced the results (Table 3 ).

In the BDNF meta-analyses [66] 83\% of patients were of Asian ethnicity, and when only white patients were studied, significant differences in AIMS scores were found between met carriers and non-met carriers (Hedges' $g=0.253$ ), there was no increase in the risk of TD. An ethnically stratified analysis may uncover group-specific effects, but to date there is no strong evidence of BDNF genetic variation driving risk of TD.

Zai et al. [67] studied the $H S P G 2$ after positive results in GWAS studies on the perlecan-coding gene and designed a methodologically robust meta-analysis (Table 3). Much of the effect seemed to originate from the original dataset and the findings could thus be more relevant in Asians, in which the original findings were found. Ethnically stratified analyses could investigate whether the HSPG2 gene plays a role in the risk of TD.

\section{Methodological issues}

In several meta-analyses studied, it became apparent that meta-analytic effects were smaller or insignificant, could not be replicated or appeared ethnically divergent. There are several possible explanations for the effects becoming smaller or insignificant. First, the so-called 'winners curse' may cause overestimation of genetic effect size in initial studies followed by low power in inconclusive follow-up studies [37]. Second, the choice of inclusion of certain studies in the meta-analyses may impact the overall outcome, for example, the inclusion of small studies or the inclusion of ethnically homogeneous or diverse studies. Third, checking for heterogeneity, publication bias, and HWE is essential for accurate results-all these factors can impact meta-analytical results. Fourth, for certain SNPs, the effect disappeared when more Asian patients were included in the analyses (e.g. Ser9Gly in DRD3). Covert ethnic stratification may exist within a defined ethnic population resulting in spurious association due to differences in allele frequencies and risk of DRMD. Association studies should be replicated controlling population stratification [68]. Fifth, in earlier studies, a higher proportion of patients were treated with FGAs than SGAs and patients may have received higher doses of AP, which may affect the rate of TD and thus increase power differentially. Finally, the concealed inclusion of more or fewer women could differentially impact the risk of TD.

The low ORs observed in the current UR are consistent with multiple genetic variants impacting complex phenotypes. To date, there has been little work in DRMD pharmacogenetics on the combined effects of multiple variations. Several reports $[69,70]$ attempted to use several genes to predict pharmacogenetic phenotypes, yielding 
some suggestive findings. However, larger patient samples are required, making this study design challenging. GWAS represent significant innovation. This technique allows hypothesis-free analyses and has shown suggestive associations with DRMD in several genes e.g. HSPG2 [53], DPP6 [30], ZNF202 [26] and eight genes in the GABAsignalling pathway [29]. Thus, the genes identified in GWASs of DRMD do not concur with candidate genes, which confirms that candidate-gene studies are prone to false-positive findings and subsequent winner's curse [71].

\section{Strengths}

The current UR has several strengths. First, A study protocol was registered on PROSPERO (study id CRD42017069634) before the reviewing process commenced [39].

Second, by judging meta-analyses by AMSTAR criteria, the most accurate quality assessment possible was achieved, e.g. judging the meta-analyses on publication bias and heterogeneity. Third, an extensive search was conducted and presumably no meta-analyses on DRMD were missed. Fourth, all references of the search were checked and selected by two authors (NB, PRB) and both authors scored the AMSTAR criteria [54]. Fifth, HWE was checked for all studies included in the UR and conclusions were made by accounting for HWE. Sixth, by conducting the current UR umbrella, meta-analyses were systematically assessed and critically compared. In this way only the highest level of evidence was assessed.

\section{Limitations}

The current UR has several limitations. First, the metaanalyses overlapped, which is not to be avoided, but must be taken into account when judging the ORs. Second, the included meta-analyses were judged by AMSTAR criteria, but AMSTAR criteria had not been established before 2014, and most of the authors could not judge the studies by formal quality assessment [72]. Therefore, several items could not be scored and AMSTAR total scores varied extensively between the included meta-analyses from " 2 " (low) to " 7 " (medium) [54]. Third, comparison between meta-analyses of the same gene was complicated because newer studies introduced ethnic groups other than White which could have resulted in covert ethnic stratification.

Pharmacogenetic studies may pave the way for personalised medicine. In clinical practice, $C Y P 2 D 6$ is already being tested to determine metabolizer status. The objective of pharmacogenetic research is to develop clinically useful genetic tests that may identify patients at high risk for adverse effects of the medication. However, research in polygenic, multifactorial inheritance needs to overcome methodological problems, e.g. sample heterogeneity, small effects of multiple genes, (epi-) genetic interactions, pleiotropy, and small sample size [73]. The modest effects of multiple genetic variants should not be underestimated, as polygenetic epigenetic effects contribute to the higher risk of TD. That is why the different polymorphisms cannot be considered individually, results are not very useful and should be interpreted with caution.

Collaboration initiatives are warranted, like large-scale programs in cancer research, which have pushed the genomics technology to new levels and have organised common standards and coordination on an international scale. This way, research overlap and redundancy can be minimised, and the value of data can be increased through comparison [74]. Progress in molecular pathology studies and their decreasing cost, increasing speed, and more comprehensive evaluation (from gene sequencing to expression profiles and proteomics) have encouraged their usage in different fields [75]. Rare variants detected by these next-generation sequencing technologies may yield a stronger signal than GWAS approaches. To date, the most modern techniques have not been used in the science of DRMD to work towards genomic personalised medicine. In the future, larger study samples are required to help find genetic information that may predict the probability of DRMD. Preferably longitudinal GWAS studies should be done which take gene-environment interactions into account and results should be placed in a DRMD database to overcome publication bias.

In conclusion, by conducting the current UR we succeeded in applying an integrated approach and in the possibility of showing effects that otherwise would not have been clear e.g. the effect of the choice of studies included in meta-analytic results. The effects of most SNPs were too small and varied to identify vulnerability for DRMD, and did not concur with GWAS work on DRMD. The UR does, however, provide clinicians with evidence-based information regarding the candidate genes that are thought to be associated with DRMD.

\section{Compliance with ethical standards}

Conflict of interest The authors declare that they have no conflict of interest.

Publisher's note Springer Nature remains neutral with regard to jurisdictional claims in published maps and institutional affiliations.

\section{References}

1. Owens DGC. A guide to the extrapyramidal side effects of antipsychotic drugs. New York: Cambridge University Press UK; 2014. 
2. Factor SA. Drug induced movement disorders malden, Mass: Blackwell Futura; 2005.

3. van Harten PN, Kahn RS. Tardive dystonia. Schizophr Bull. 1999;25:741-8.

4. Lambert M, Conus P, Eide P, Mass R, Karow A, Moritz S, et al. Impact of present and past antipsychotic side effects on attitude toward typical antipsychotic treatment and adherence. Eur Psychiatry. 2004;19:415-22.

5. Casey DE. Implications of the CATIE trial on treatment: extrapyramidal symptoms. CNS Spectr. 2006;11:25-31.

6. Correll CU, Schenk EM. Tardive dyskinesia and new antipsychotics. Curr Opin Psychiatry. 2008;21:151-6.

7. Taylor D. Antipsychotic polypharmacy-confusion reigns. Psychiatrist; 2010;34:41-43.

8. Mentzel TQ, Lieverse R, Bloemen O, Viechtbauer W, van Harten PN, Genetic Risk and Outcome of Psychosis (GROUP) Investigators. et al. High incidence and prevalence of drug-related movement disorders in young patients with psychotic disorders. J Clin Psychopharmacol. 2017;37:231-8.

9. Bakker PR, de Groot IW, van Os J, van Harten PN. Long-stay psychiatric patients: a prospective study revealing persistent antipsychotic-induced movement disorder. PLoS ONE. 2011;6: e25588.

10. van Harten PN, Matroos GE, Hoek HW, Kahn RS. The prevalence of tardive dystonia, tardive dyskinesia, parkinsonism and akathisia The Curacao Extrapyramidal Syndromes Study: I. Schizophr Res. 1996;19:195-203.

11. Glazer WM, Morgenstern H, Doucette J. Race and tardive dyskinesia among outpatients at a CMHC. Hosp Community Psychiatry. 1994;45:38-42.

12. Halstead SM, Barnes TR, Speller JC. Akathisia: prevalence and associated dysphoria in an in-patient population with chronic schizophrenia. Br J Psychiatry. 1994;164:177-83.

13. Sachse C, Brockmoller J, Bauer S, Roots I. Functional significance of a $\mathrm{C}->\mathrm{A}$ polymorphism in intron 1 of the cytochrome P450 CYP1A2 gene tested with caffeine. Br J Clin Pharm. 1999;47:445-9.

14. Sachdev PS. Neuroleptic-induced movement disorders: an overview. Psychiatr Clin North Am. 2005;28:255-74.

15. Tenback DE, van Harten PN, van Os J. Non-therapeutic risk factors for onset of tardive dyskinesia in schizophrenia: a metaanalysis. Mov Disord. 2009;24:2309-15.

16. Leucht S, Corves C, Arbter D, Engel RR, Li C, Davis JM. Second-generation versus first-generation antipsychotic drugs for schizophrenia: a meta-analysis. Lancet. 2009;373:31-41.

17. Fujimaki K, Morinobu S, Yamashita H, Takahashi T, Yamawaki S. Predictors of quality of life in inpatients with schizophrenia. Psychiatry Res. 2012;197:199-205.

18. Schouten HJ, Knol W, Egberts TC, Schobben AF, Jansen PA, van Marum RJ. Quality of life of elderly patients with antipsychoticinduced parkinsonism: a cross-sectional study. J Am Med Dir Assoc. 2012;13:82 e81-85.

19. Haddad PM, Dursun SM. Neurological complications of psychiatric drugs: clinical features and management. Hum Psychopharmacol. 2008;23:15-26. Jan

20. Ballesteros J, Gonzalez-Pinto A, Bulbena A. Tardive dyskinesia associated with higher mortality in psychiatric patients: results of a meta-analysis of seven independent studies. J Clin Psychopharmacol. 2000;20:188-94.

21. Chong SA, Tay JA, Subramaniam M, Pek E, Machin D. Mortality rates among patients with schizophrenia and tardive dyskinesia. J Clin Psychopharmacol. 2009;29:5-8.

22. Dean CE, Thuras PD. Mortality and tardive dyskinesia: long-term study using the US National Death Index. Br J Psychiatry. 2009;194:360-4.
23. Suzuki M, Hurd YL, Sokoloff P, Schwartz JC, Sedvall G. D3 dopamine receptor mRNA is widely expressed in the human brain. Brain Res. 1998;779:58-74.

24. Accili D, Fishburn CS, Drago J, Steiner H, Lachowicz JE, Park $\mathrm{BH}$, et al. A targeted mutation of the D3 dopamine receptor gene is associated with hyperactivity in mice. Proc Natl Acad Sci USA. 1996;93:1945-9.

25. GCCC Psychiatric, Cichon S, Craddock N, Daly M, Faraone SV, Gejman PV, et al. Genomewide association studies: history, rationale, and prospects for psychiatric disorders. Am J Psychiatry. 2009;166:540-56.

26. Aberg K, Adkins DE, Bukszár J, Webb BT, Caroff SN, Miller $\mathrm{DD}$, et al. Genomewide association study of movement-related adverse antipsychotic effects. Biol Psychiatry. 2010;67:279-82.

27. Alkelai A, Greenbaum L, Rigbi A, Kanyas K, Lerer B. Genomewide association study of antipsychotic-induced parkinsonism severity among schizophrenia patients. Psychopharmacol (Berl). 2009;206:491-9.

28. Greenbaum L, Alkelai A, Rigbi A, Kohn Y, Lerer B. Evidence for association of the GLI2 gene with tardive dyskinesia in patients with chronic schizophrenia. Mov Disord. 2010;25:2809-17.

29. Inada T, Koga M, Ishiguro H, Horiuchi Y, Syu A, Yoshio T, et al. Pathway-based association analysis of genome-wide screening data suggest that genes associated with the gamma-aminobutyric acid receptor signaling pathway are involved in neurolepticinduced, treatment-resistant tardive dyskinesia. Pharmacogenet Genomics. 2008;18:317-23.

30. Tanaka S, Syu A, Ishiguro H, Inada T, Horiuchi Y, Ishikawa M, et al. DPP6 as a candidate gene for neuroleptic-induced tardive dyskinesia. Pharmacogenomics J. 2013;13:27-34.

31. Reynolds GP. The impact of pharmacogenetics on the development and use of antipsychotic drugs. Drug Disco Today. 2007;12:953-9.

32. Ohmori O, Shinkai T, Hori H, Matsumoto C, Nakamura J. A perspective on molecular genetic studies of tardive dyskinesia: one clue for individualized antipsychotic drug therapy. Prog Neuropsychopharmacol Biol Psychiatry. 2003;27:581-6.

33. Lerer B, Segman RH. Pharmacogenetics of antipsychotic therapy: pivotal research issues and the prospects for clinical implementation. Dialogues Clin Neurosci. 2006;8:85-94.

34. Lencz T, Malhotra AK. Pharmacogenetics of antipsychoticinduced side effects. Dialogues Clin Neurosci. 2009;11:405-15.

35. Zhang JP, Malhotra AK. Pharmacogenetics and antipsychotics: therapeutic efficacy and side effects prediction. Expert Opin Drug Metab Toxicol. 2011;7:9-37.

36. Lanning RK, Zai CC, Muller DJ. Pharmacogenetics of tardive dyskinesia: an updated review of the literature. Pharmacogenomics. 2016;17:1339-51.

37. Xiao R, Boehnke M. Quantifying and correcting for the winner's curse in genetic association studies. Genet Epidemiol. 2009;33:453-62.

38. Poirier JG, Faye LL, Dimitromanolakis A, Paterson AD, Sun L, Bull SB. Resampling to address the winner's curse in genetic association analysis of time to event. Genet Epidemiol. 2015;39:518-28.

39. PROSPERO. The genetics of drug-related movement disordersan umbrella review of meta analyses; 2017. https://www.crd.york. ac.uk/PROSPERO/display_record.php?RecordID $=69634$.

40. Bakker PR, van Harten PN, van Os J. Antipsychotic-induced tardive dyskinesia and polymorphic variations in COMT, DRD2, CYP1A2 and MnSOD genes: a meta-analysis of pharmacogenetic interactions. Mol Psychiatry. 2008;13:544-56.

41. Fleeman N, Dundar Y, Dickson R, Jorgensen A, Pushpakom S, McLeod C, et al. Cytochrome P450 testing for prescribing antipsychotics in adults with schizophrenia: systematic review and meta-analyses. Pharmacogenomics J. 2011;11:1-14. 
42. Patsopoulos NA, Ntzani EE, Zintzaras E, Ioannidis JP. CYP2D6 polymorphisms and the risk of tardive dyskinesia in schizophrenia: a meta-analysis. Pharmacogenet Genomics. 2005;15:151-8.

43. Ozdemir V, Aklillu E, Mee S, Bertilsson L, Albers LJ, Graham JE, et al. Pharmacogenetics for off-patent antipsychotics: reframing the risk for tardive dyskinesia and access to essential medicines. Expert Opin Pharmacother. 2006;7:119-33.

44. Lerer B, Segman RH, Tan EC. Pharmacogenetics of psychotropic drugs. 2002 ed. Cambridge: Cambridge University Press; 2002.

45. Bakker PR, van Harten PN, van Os J. Antipsychotic-induced tardive dyskinesia and the Ser9Gly polymorphism in the DRD3 gene: a meta analysis. Schizophr Res. 2006;83:185-92.

46. Neville MJ, Johnstone EC, Walton RT. Identification and characterization of ANKK1: a novel kinase gene closely linked to DRD2 on chromosome band 11q23.1. Hum Mutat. 2004;23:540-5.

47. Zai CC, De Luca V, Hwang RW, Voineskos A, Muller DJ, Remington G, et al. Meta-analysis of two dopamine D2 receptor gene polymorphisms with tardive dyskinesia in schizophrenia patients. Mol Psychiatry. 2007;12:794-5.

48. Cadet JL, Lohr JB. Possible involvement of free radicals in neuroleptic-induced movement disorders. Evidence from treatment of tardive dyskinesia with vitamin E. Ann N Y Acad Sci. 1989;570:176-85.

49. Patsopoulos NA, Ntzani EE, Zintzaras E, Ioannidis JP. CYP2D6 polymorphisms and the risk of tardive dyskinesia in schizophrenia: a meta-analysis. Pharmacogenet Genomics. 2005;15:151-158. https://doi.org/10.1097/01213011-200503000-00003.

50. Park H, Poo MM. Neurotrophin regulation of neural circuit development and function. Nat Rev Neurosci. 2013;14:7-23.

51. Egan MF, Kojima M, Callicott JH, Goldberg TE, Kolachana BS, Bertolino A, et al. The BDNF val66met polymorphism affects activity-dependent secretion of BDNF and human memory and hippocampal function. Cell. 2003;112:257-69.

52. Nicole S, Davoine CS, Topaloglu H, Cattolico L, Barral D, Beighton P, et al. Perlecan, the major proteoglycan of basement membranes, is altered in patients with Schwartz-Jampel syndrome (chondrodystrophic myotonia). Nat Genet. 2000;26:480-3.

53. Syu A, Ishiguro H, Inada T, Horiuchi $Y$, Tanaka S, Ishikawa M, et al. Association of the HSPG2 gene with neuroleptic-induced tardive dyskinesia. Neuropsychopharmacology. 2010;35:1155-64.

54. Shea BJ, Grimshaw JM, Wells GA, Boers M, Andersson N, Hamel C, et al. Development of AMSTAR: a measurement tool to assess the methodological quality of systematic reviews. BMC Med Res Methodol. 2007;7:10.

55. Namipashaki A, Razaghi-Moghadam Z, Ansari-Pour N. The essentiality of reporting hardy-weinberg equilibrium calculations in population-based genetic association studies. Cell J Summer. 2015;17:187-92.

56. Lerer B, Segman RH, Fangerau H, Daly AK, Basile VS, Cavallaro $\mathrm{R}$, et al. Pharmacogenetics of tardive dyskinesia: combined analysis of 780 patients supports association with dopamine D3 receptor gene Ser9Gly polymorphism. Neuropsychopharmacology. 2002;27:105-19.

57. Utsunomiya K, Shinkai T, Sakata S, Yamada K, Chen HI, De Luca V, et al. Genetic association between the dopamine D3 receptor gene polymorphism (Ser9Gly) and tardive dyskinesia in patients with schizophrenia: a reevaluation in East Asian populations. Neurosci Lett. 2012;507:52-56.

58. Tsai HT, North KE, West SL, Poole C. The DRD3 rs6280 polymorphism and prevalence of tardive dyskinesia: a meta-analysis. Am J Med Genet B Neuropsychiatr Genet. 2010;153B:57-66.
59. Zai CC, Tiwari AK, Müller DJ, De Luca V, Shinkai T, Shaikh S, et al. The catechol-O-methyl-transferase gene in tardive dyskinesia. World J Biol Psychiatry. 2010;11:803-12.

60. Lv Z, Rong B, Tong X, Li X, Chen X, Wang X, et al. The association between COMT Val158Met gene polymorphism and antipsychotic-induced tardive dyskinesia risk. Int $\mathrm{J}$ Neurosci. 2016; 126:1044-50.

61. Lerer B, Segman RH, Tan EC, Basile VS, Cavallaro R, Aschauer $\mathrm{HN}$, et al. Combined analysis of 635 patients confirms an agerelated association of the serotonin $2 \mathrm{~A}$ receptor gene with tardive dyskinesia and specificity for the non-orofacial subtype. Int $\mathbf{J}$ Neuropsychopharmacol. 2005;8:411-25.

62. Galecki P, Pietras T, Szemraj J. Manganese superoxide dismutase gene (MnSOD) polimorphism in schizophrenics with tardive dyskinesia from central Poland. Psychiatr Pol. 2006;40:937-48.

63. Pae CU, Yoon SJ, Patkar A, Kim JJ, Jun TY, Lee C, et al. Manganese superoxide dismutase (MnSOD: Ala-9Val) gene polymorphism and mood disorders: a preliminary study. Prog Neuropsychopharmacol Biol Psychiatry. 2006;30:1326-9.

64. Zai CC, Tiwari AK, Basile V, de Luca V, Müller DJ, Voineskos $\mathrm{AN}$, et al. Oxidative stress in tardive dyskinesia: genetic association study and meta-analysis of NADPH quinine oxidoreductase 1 (NQO1) and Superoxide dismutase 2 (SOD2, MnSOD) genes. Prog Neuropsychopharmacol Biol Psychiatry. 2010;34:50-56.

65. Wang DF, Cao B, Xu MY, Liu YQ, Yan LL, Liu R, et al. Metaanalyses of manganese superoxide dismutase activity, gene Ala$9 \mathrm{Val}$ polymorphism, and the risk of schizophrenia. Med (Baltim). 2015;94:e1507.

66. Miura I, Zhang JP, Nitta M, Lencz T, Kane JM, Malhotra AK, et al. BDNF Val66Met polymorphism and antipsychotic-induced tardive dyskinesia occurrence and severity: a meta-analysis. Schizophr Res. 2014;152:365-72.

67. Zai CC, Lee FH, Tiwari AK, Lu JY, de Luca V, Maes MS, et al. Investigation of the HSPG2 gene in tardive dyskinesia-new data and meta-analysis. Front Pharm. 2018;9:974.

68. Hellwege JN, Keaton JM, Giri A, Gao X, Velez Edwards DR, Edwards TL. Population stratification in genetic association studies. Curr Protoc Hum Genet. 2017;95:1-22.

69. Segman RH, Heresco-Levy U, Finkel B, Goltser T, Shalem R, Schlafman M, et al. Association between the serotonin 2A receptor gene and tardive dyskinesia in chronic schizophrenia. Mol Psychiatry. 2001;6:225-9.

70. Basile VS, Masellis M, Potkin SG, Kennedy JL. Pharmacogenomics in schizophrenia: the quest for individualized therapy. Hum Mol Genet. 2002;11:2517-30.

71. Farrell MS, Werge T, Sklar P, Owen MJ, Ophoff RA, O'Donovan $\mathrm{MC}$, et al. Evaluating historical candidate genes for schizophrenia. Mol Psychiatry. 2015;20:555-62.

72. Wells G, Shea B, O'Connell D, Peterson J, Welch V, Losos M, et al. The Newcastle-Ottawa Scale (NOS) for assessing the quality of nonrandomised studies in meta-analyses. 2013. Available at: http://www.ohri.ca/programs/clinical_epidemiology/oxford.asp.

73. Abdolmaleky HM, Thiagalingam S, Wilcox M. Genetics and epigenetics in major psychiatric disorders: dilemmas, achievements, applications, and future scope. Am J Pharmacogenomics. 2005;5:149-60.

74. Chin L, Andersen JN, Futreal PA. Cancer genomics: from discovery science to personalized medicine. Nat Med. 2011;17:297-303.

75. Tannock IF, Hickman JA. Limits to personalized cancer medicine. N. Engl J Med. 2016;375:1289-94. 